\title{
Developmental, morphological and molecular variation of commercial Ganoderma spp. accessions from southern Vietnam
}

\author{
VIET THE HO ${ }^{1, \bullet}$, THI NGOC HA VO ${ }^{1,2}$, NGOC GIAU LE ${ }^{1}$ \\ ${ }^{1}$ Ho Chi Minh City University of Food Industry. 140 Le Trong Tan Street, Tan Phu District, Ho Chi Minh City, Vietnam. •email: thehv@ hufi.edu.vn \\ ${ }^{2}$ Tien Giang Applied Science and Technology Research Center. 555 Ap Bac, Ward 5, My Tho City, Tien Giang Province, Vietnam
}

Manuscript received: 7 November 2019. Revision accepted: 23 November 2019.

\begin{abstract}
Ho VT, Vo TNH, Le NG. 2019. Developmental, morphological and molecular variation of commercial Ganoderma spp. accessions from southern Vietnam. Biodiversitas 20: 3684-3689. In Vietnam, the market value of Ganoderma spp. increases markedly recently due to the high demand as an effective medicine. However, the breeding programs for this mushroom are still less effective due to the poor knowledge of genetic composition. This study was conducted to investigate the diversity of some commercial Ganoderma spp. accessions from southern Vietnam using both traditional techniques using developmental and morphological characterization, and molecular method using ISSR markers. A total of nine Ganoderma spp. samples were collected from production areas and research institutes in southern provinces of Vietnam and targeted for developmental and molecular characterization. Developmental and morphological variations among accessions were evaluation. In addition, 20 ISSR markers were also utilized for further understanding genetic composition of these mushrooms. The obtained measurement data show a large variation among the studied Ganoderma spp. accessions in term of mycelia growth, diameter, shape, and dried weight of fruiting bodies. Molecular data from 20 ISSR markers also supported genetic variation of the examined samples. Based on the results of this study, the samples of Ganoderma spp. accessions from southern Vietnam can be divided into two main groups which are not correlated to geographical locations of sample collection sites. The results are potential for classification and provide new insights for molecular assisted selection of Ganoderma spp.
\end{abstract}

Keywords: Development, Ganoderma, ISSR, molecular markers, morphology

\section{INTRODUCTION}

The production of Ganoderma spp. (or lingzhi in Vietnamese) increases sharply in Vietnam recently. This mushroom gets public attention due to its high medical values, such as anti-tumor, anti-inflammatory, antiviral (e.g. anti-HIV) and antibacterial activity, as well as to lower blood pressure, cholesterol, and blood sugar levels (Bao et al. 2002). Due to out-crossing over generations and different origins, the genetic composition of this mushroom is highly heterogeneous, leading to a large developmental and morphological variation even within the same species (Hong et al. 2004). Furthermore, several efforts have been performed in Ganoderma spp. breeding which resulted in several cultivars has been marketed leading to difficulty in identification. Morphological characterization has been widely applied when identifying Ganoderma, yet this method is sensitive to environmental and physiological variations (Staniaszek et al. 2002; Iqbal et al. 2010).

Recently, molecular markers have been utilized to identify different species widely because of numerous advantages, such as unlimited in marker number, unaffected by environment and growing conditions, and easy to interpret with reliable repeatable results. Several molecular markers have been developed, for instance isozyme, Restriction Fragment Length Polymorphism (RFLP), Random Amplified of Polymorphic DNA (RAPD), Amplified Fragment Length Polymorphism (AFLP), Simple Sequence Repeat (SSR), Inter-Simple
Sequence Repeat (ISSR), and Single Nucleotide Polymorphisms (SNP). Among them, ISSR markers are highly preferred because they are based on Polymerase Chain Reaction (PCR) markers and possess common advantages, such as they are simple, rapid and low cost, require minimum laboratory skill, require small DNA quantity, accommodate high number of fragments in each reaction, and do not require prior knowledge of genetic genome of targeted plants. Furthermore, ISSR is a preferred method as a highly variable, reproducible marker (Wolfe and Liston 1998) leading to expanded use in genetic diversity research, population genetic studies, genetic markers, crop identification, source analysis, identification, genetic change identification, and crossbreeding in different fungus species, such as Piptoporus betulinus (Jing et al. 2017), Lepista nuda (Du et al. 2018), and Colletotrichum falcatum Went (Patel et al. 2018) . Nevertheless, ISSR markers have not been commonly used for genetic characterization of Ganoderma. This study was conducted to investigate the diversity of some commercial Ganoderma spp. accessions from southern Vietnam using both traditional techniques using developmental and morphological characterization, and molecular method using ISSR markers. The obtained results could provide scientific information for classification, identification, and authentication of Ganoderma spp. in Vietnam. 


\section{MATERIALS AND METHODS}

\section{Sample collection}

Ganoderma spp. samples were collected from research institutes, universities, and companies in different provinces in southern Vietnam (Figure 1 and Table 1). After sampling, samples were grown on solid PDA (potato, glucose, and agar) medium, and the culture plates were incubated at room temperature to allow mycelia ramification. The growth of secondary mycelia was recorded at 4, 8 and 12 days after inoculation (DAI). The grain spawns were inoculated in steamed-sterilized fruiting bags containing rubbery tree-sawdust $(97 \%)+$ cornflour $(2 \%)+$ rice bran $(1 \%)$ then stored at room temperature until the mycelia colonized the bags fully. Then, the bags were opened to allow the development of primordia and fruiting body maturity. Developmental and morphological characteristics including dry weight, shape, diameter, and thickness of fruiting body were observed and recorded. The experiments were followed by Randomized Complete Block design with 4 replications. The data was analyzed using Statgraphics Centurion XV.1 software (Statgraphics Technologies, Inc.). The differences between survey samples were tested by Analysis of Variance (ANOVA). The mean values were verified by Duncan test at significance level of $5 \%$.

\section{DNA extraction}

Total DNA was extracted from Ganoderma spp. mycelia using the method described by Porebski and colleagues (Porebski et al. 1997). DNA quality was then tested by electrophoresis on $1 \%$ agarose gel in TAE $1 \mathrm{X}$ buffer and stained with Gelred dye (Biotium, USA). The result was observed under ultraviolet light by Quantum ST4 3000 gel reader (Montreal - Biotech, Canada). DNA concentrations were determined by spectrophotometer (Optima SP 3000 nano UV-VIS, Japan).

\section{ISSR reaction}

A total of 20 ISSR primers (Levi et al. 2004) were used and shown in Table 2. The composition of PCR reactions was performed as follows: $7.5 \mu \mathrm{L} 2 \mathrm{X}$ Mytaq Red Mix (Bioline, UK), 20 ng DNA, $0.2 \mu \mathrm{M}$ primer and PCR water for final volume of $15 \mu \mathrm{l}$. The ISSR reaction conditions were conducted as follows: initial denaturation at $95^{\circ} \mathrm{C}$ for 2 minutes; then 35 cycles of 30 seconds at $95{ }^{\circ} \mathrm{C}, 30$ seconds at $54{ }^{\circ} \mathrm{C}$, and 1 minute at $72{ }^{\circ} \mathrm{C}$. Finally, 5 minutes was added to finish reaction at $72{ }^{\circ} \mathrm{C}$. All reactions were carried out with the SureCycler 8800 Thermal Cycler (Agilent, USA). PCR amplification was then separated by electrophoresis in $1.5 \%$ agarose gel in $1 \mathrm{X}$ TAE buffer, and stained with $0.5 \mu \mathrm{g} / \mathrm{ml}$ Gelred TM loading buffer then visualized under UV transilluminator (Quantum - ST4 3000, Montreal - Biotech, Canada).

\section{Data analysis}

After gel electrophoresis, the clear amplification bands were used in the analysis. Only clear bands were analyzed; weak signal bands were excluded from final analysis. Clearly visible ISSR amplified bands were scored as 1, whereas the absent band was scored as 0 . Phylogeny is built based on the Unweighted Pair Group Method with the Arithmetic mean (UPGMA) and the algorithm with the SAHN module in NTSYSpc 2.1 (Rohlf 1987).

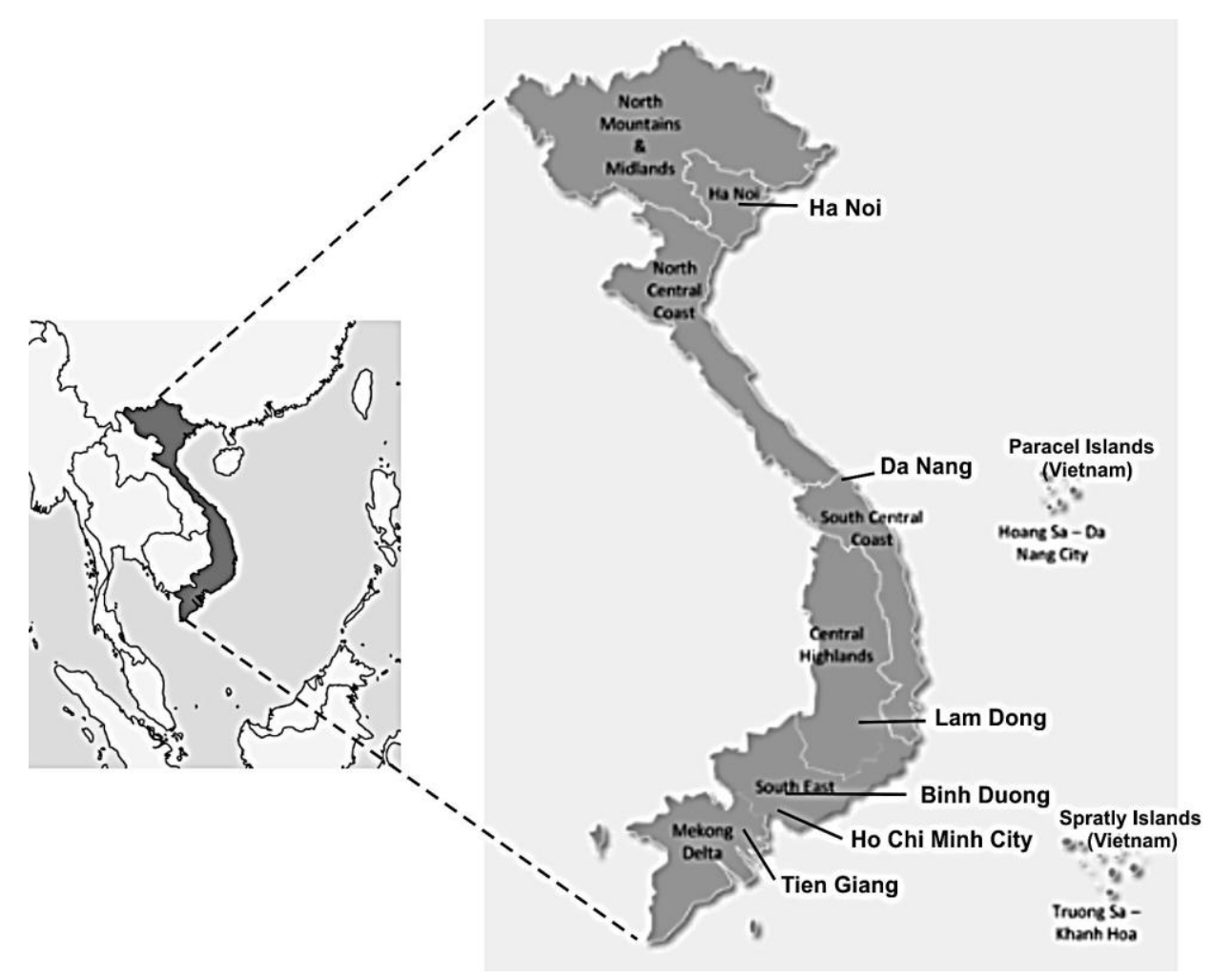

Figure 1. Targeted areas for collecting Ganoderma spp. accessions in this study 
Table 1: Collection sites of nine Ganoderma spp. accessions used in the study

\begin{tabular}{ll}
\hline Collection site & Sample code \\
\hline Applied Science and Technology Research Center, Tien Giang province & TG1 \\
Applied Science and Technology Research Center, Tien Giang province & TG2 \\
Tan Hung village, Tan Thoi commune, Tan Phu Dong district, Tien Giang province & TG3 \\
Lam Dong Center for Science and Technology Application & LD \\
Healthy Fungi Company Limited, Da Nang city & DN \\
Hamlet 5, Binh My commune, Cu Chi district, City. Ho Chi Minh & CC \\
Dinh Hoa Ward, Thu Dau Mot city, Binh Duong province & BD1 \\
Experimental Research Center of Thu Dau Mot University, Binh Duong province & BD2 \\
Center for Mushroom Research and Development, Tu Liem District, Hanoi & HN \\
\hline
\end{tabular}

\section{RESULTS AND DISCUSSION}

\section{Morphological characterization}

There was a large variation in mycelium growth of the nine Ganoderma samples (Table 3). The TG1 and BD1 samples showed the fastest growth from 4 days until 12 days after inoculation. Whereas, some genotypes had slow adaptation to growing medium at 4 days but grew faster in period of 12 days, such as the BD2 and TG3. The growth time of the mycelium is very important and plays a decisive role in the growth, development of fruiting bodies and the yield of Ganoderma. Because this indicator can affect the disease resistance of fungal strains. Based on the speed of spreading mycelium, growers can adjust the time and watering regime accordingly, contributing to the increase in mushroom yield.

Pileus shape is important characteristic of Ganoderma fruiting body since it affects the commercial value of this mushroom. The nine samples in this study also showed the variation in pieus shape as shown in Figure 2.

Liu and colleagues suggested the division of Ganoderma pieus shape into three groups, namely kidney type, flabelliform type and anther type (Liu et al. 2017). Among the nine samples, the $\mathrm{CC}$ sample collected from $\mathrm{Cu}$ Chi district of Ho Chi Minh city showed the most distinct shape. The fruit consisted of several combining small ears and shaped like a deer horn. Due to the shape of a deer horn, this genotype is suitable to grow as ornamental plant and bonsai. The samples TG1, BD1, BD2, and TG3 showed similar shapes of the fruiting body which were alike kidney-shaped. The remaining samples TG2, LD, DN, HN had different shapes of kidney, slightly skewed ear and fan-shaped.

There was a difference in the thickness of the fruiting bodies of the studied samples (Table 4). Thickness ranged from 1.10 to $1.60 \mathrm{~cm}$. The sample with the largest thickness of fungal ear was LD $(1.60 \mathrm{~cm})$ and the lowest thickness was DN $(1.04 \mathrm{~cm})$ and $\mathrm{HN}(1.10 \mathrm{~cm})$. The canopy diameter also varied with the BD1 and TG2 genotypes had the largest canopy diameter, with 11.70 and $11.90 \mathrm{~cm}$, respectively. Whereas, the sample with the smallest canopy diameter was the CC sample $(5.01 \mathrm{~cm})$. The result of this study is in agreement with study of Le and Tran conducted in Ganoderma samples from Thua Thien Hue- a central province of Vietnam- in 2008 (Le and Tran 2008).
There was also large variation in dried weight of fruiting body among genotypes. The CC and TG2 also showed the highest dried weight with $19.05 \mathrm{~g}$ and $17.68 \mathrm{~g}$, respectively. Samples collected from Lam Dong province (LD), Da Nang province (DN), and Ha Noi (HN) varied from $5.69 \mathrm{~g}$ to $7.23 \mathrm{~g}$.

Table 2: Sequences of 20 ISSR markers in this study.

\begin{tabular}{ll}
\hline Primer & Sequence 5'- $\mathbf{3}$ \\
\hline UBC880 & GGAGAGGAGAGGAGA \\
UBC825 & ACACACACACACACACT \\
UBC841 & GAGAGAGAGAGAGAGACTC \\
UBC855 & ACACACACACACACACCTT \\
UBC813 & CTCTCTCTCTCTCTCTT \\
UBC853 & TCTCTCTCTCTCTCTCRT \\
UBC809 & AGAGAGAGAGAGAGAGG \\
UBC814 & CTCTCTCTCTCTCTCTA \\
UBC811 & GAGAGAGAGAGAGAGAC \\
UBC810 & GAGAGAGAGAGAGAGAT \\
UBC808 & AGAGAGAGAGAGAGAGAG \\
UBC814- 11 & CTCTCTCTCTCTCTCAT \\
UBC818 & CACACACACACACACAG \\
UBC820 & GTGTGTGTGTGTGTGTC \\
UBC826 & ACACACACACACACACC \\
UBC826- 11 & ACACACACACACACACC \\
UBC834 & AGAGAGAGAGAGAGACYT \\
UBC848 & CACACACACACACACARG \\
UBC854 & TCTCTCTCTCTCTCTCAGG \\
UBC856 & ACACACACACACACACCTA \\
\hline
\end{tabular}

Table 3. Mycelium development of nine Ganoderma accessions at different time points.

\begin{tabular}{llll}
\hline Sample & $\begin{array}{l}\mathbf{4} \text { DAI } \\
(\mathbf{c m})\end{array}$ & $\begin{array}{l}\mathbf{8} \mathbf{D A I} \\
(\mathbf{c m})\end{array}$ & $\begin{array}{l}\mathbf{1 2} \mathbf{D A I} \\
(\mathbf{c m})\end{array}$ \\
\hline TG1 & $1,90^{\mathrm{a}}$ & $5,54^{\mathrm{a}}$ & $11,66^{\mathrm{a}}$ \\
TG2 & $1,44^{\text {cde }}$ & $3,86^{\mathrm{b}}$ & $7,74^{\mathrm{b}}$ \\
LD & $1,24^{\mathrm{eg}}$ & $3,42^{\mathrm{bc}}$ & $7,00^{\mathrm{bc}}$ \\
DN & $1,30^{\mathrm{deg}}$ & $3,46^{\mathrm{bc}}$ & $7,32^{\mathrm{b}}$ \\
CC & $1,52^{\mathrm{cd}}$ & $3,64^{\mathrm{b}}$ & $7,18^{\mathrm{b}}$ \\
BD1 & $1,92^{\mathrm{a}}$ & $5,96^{\mathrm{a}}$ & $12,24^{\mathrm{a}}$ \\
BD2 & $1,64^{\mathrm{bc}}$ & $5,54^{\mathrm{a}}$ & $11,48^{\mathrm{a}}$ \\
TG3 & $1,80^{\mathrm{ab}}$ & $5,50^{\mathrm{a}}$ & $11,54^{\mathrm{a}}$ \\
HN & $1,50^{\mathrm{cd}}$ & $3,54^{\mathrm{b}}$ & $6,86^{\mathrm{bc}}$ \\
\hline
\end{tabular}

Note: Means within a column having the different letter of superscript are significantly different from each other at 0.05 level of significance using Duncan test (DAI: days after inoculum). 


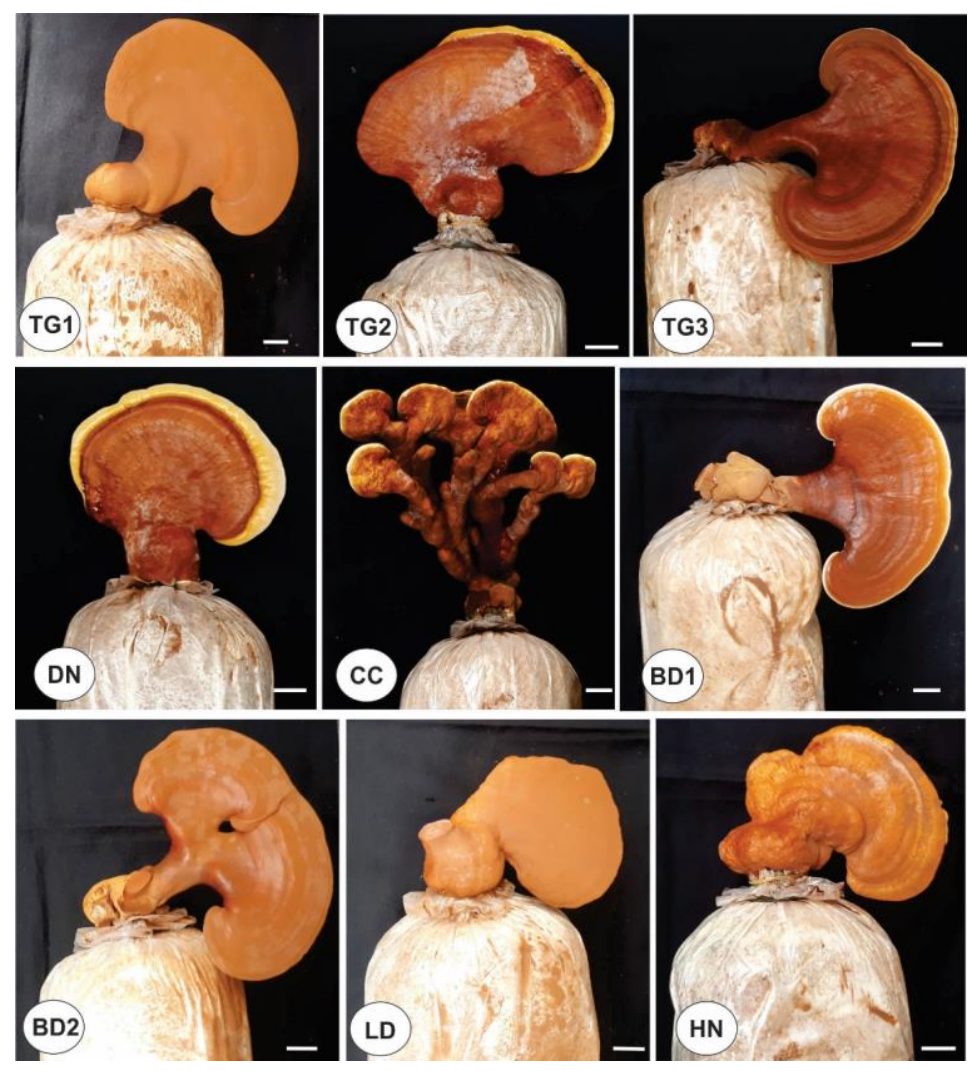

Figure 2. Morphology of nine Ganoderma spp. accessions used in this study. (The bar is equal to $2 \mathrm{~cm}$ )

\section{ISSR analysis}

ISSR markers have been successfully used to study genetic composition of different fungus species (Jing et al. 2017; Du et al. 2018; Patel et al. 2018) except Ganoderma. In this study, the potential ability of ISSR markers was used to examine the genetic diversity of nine popular Ganoderma genotypes collected from different provinces in southern Vietnam. Twenty ISSR primers were used to evaluate the genetic composition of nine Ganoderma genotypes, and the tested primers showed clear and reproducible bands (Figure 3 ).

A total of 244 bands were generated in which 242 bands were polymorphic, accounting for $99.25 \%$. The size of amplified bands ranged from 200 to $3000 \mathrm{bps}$ (Table 5). The number of bands varied from 8 (primer UBC834) to 16 (primer UBC854). The average of amplified bands and polymorphic bands per primer were 14.2 and 14.1, respectively. Polymorphic percentage ranged between 92.3\% (primer UBC826- 11) to $100 \%$ (primer UBC880; UBC841; UBC855; UBC813; UBC853; UBC809; UBC814; UBC811; UBC810; UBC808; UBC814-11; UBC818; UBC820; UBC826; UBC834; UBC848; UBC854; and UBC856), with an average of 99.25\%. ISSR primers showed high PIC values from 0.73 (primer UBC880) to 0.94 (primer UBC848) with average of 0.85 meaning that all these primers are suitable for studying genetic diversity in Ganoderma according to Botstein et al. (1980). The results showed that the complex marker patterns of ISSR will be advantageous for distinguishing closely related species (Jaipreet et al. 2015).
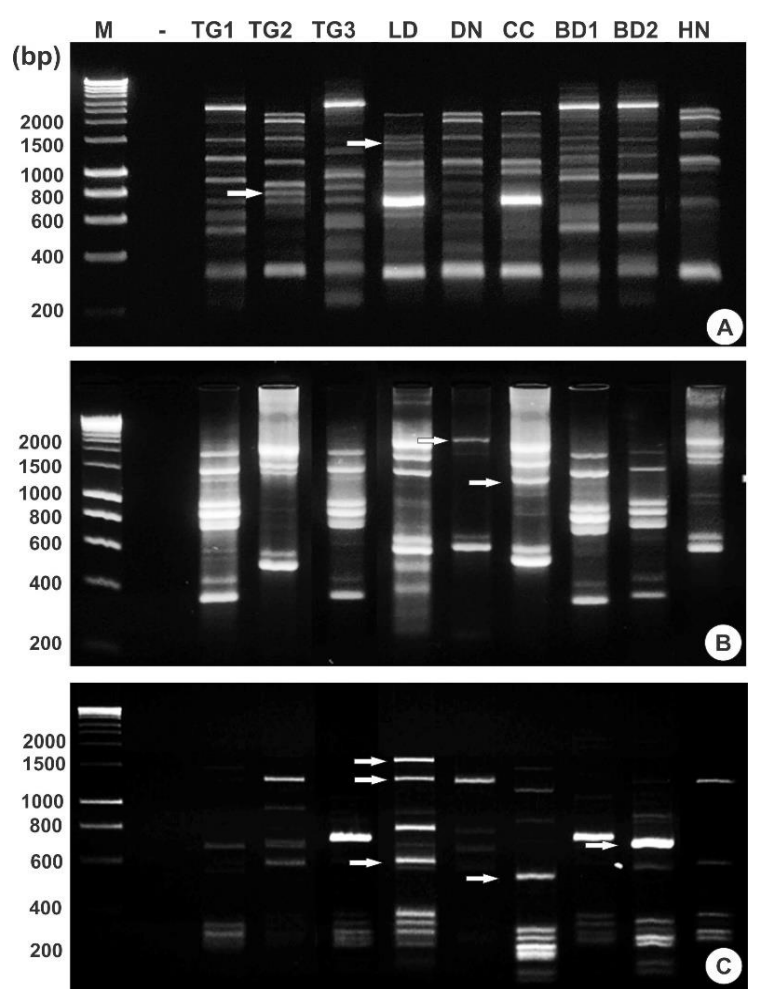

Figure 3. Representative ISSR results of nine Ganoderma genotypes with UBC853primer (A), UBC825 primer (B), and UBC814 (C). (The abbreviations are corresponding to sample number in Table 1; M: DNA marker; -: Negative control without DNA in reaction. The white arrows present the bands which are potential for genotype identification) 


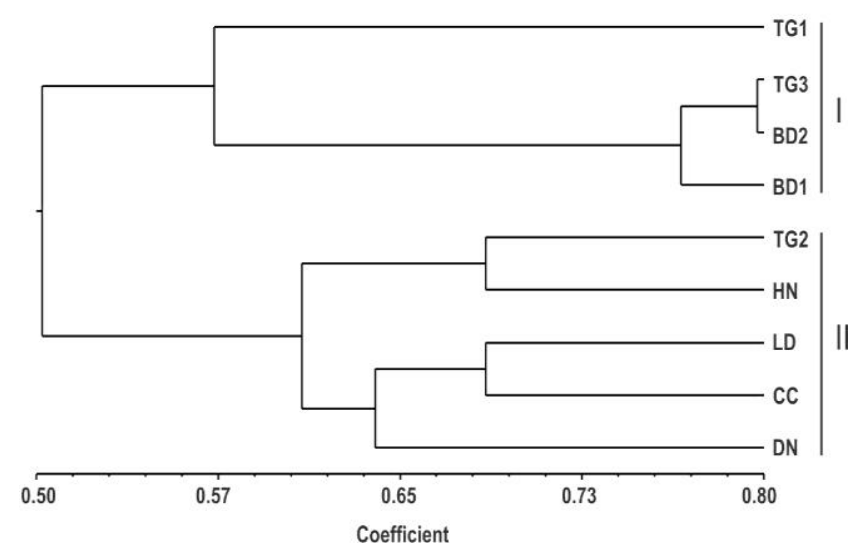

Figure 4. Dendrogram obtains from nine genotypes of Ganoderma genotypes collected in Vietnam with UPGMA based on Jaccard's coefficient by using 20 ISSR primers.

Table 4. Morphological variation in fruiting body of nine Ganoderma spp. accessions

\begin{tabular}{llll}
\hline Sample & $\begin{array}{l}\text { Thickness } \\
(\mathbf{c m})\end{array}$ & $\begin{array}{c}\text { Diameter } \\
(\mathbf{c m})\end{array}$ & $\begin{array}{l}\text { Dried weight } \\
(\mathbf{g})\end{array}$ \\
\hline TG1 & $1.29^{\mathrm{b}}$ & $10.31^{\mathrm{c}}$ & $12.54^{\mathrm{b}}$ \\
TG2 & $1.29^{\mathrm{b}}$ & $11,90^{\mathrm{a}}$ & $17,68^{\mathrm{a}}$ \\
LD & $1.60^{\mathrm{a}}$ & $10.36^{\mathrm{bc}}$ & $7.23^{\mathrm{c}}$ \\
DN & $1.04^{\mathrm{c}}$ & $7.44^{\mathrm{d}}$ & $5.69^{\mathrm{c}}$ \\
CC & $1.31^{\mathrm{b}}$ & $5.01^{\mathrm{e}}$ & $19.05^{\mathrm{a}}$ \\
BD1 & $1.27^{\mathrm{b}}$ & $11.70^{\mathrm{a}}$ & $12.41^{\mathrm{b}}$ \\
BD2 & $1.33^{\mathrm{b}}$ & $11.06^{\mathrm{abc}}$ & $12.79^{\mathrm{b}}$ \\
TG3 & $1.35^{\mathrm{b}}$ & $11.30^{\mathrm{ab}}$ & $12,99^{\mathrm{b}}$ \\
HN & $1.10^{\mathrm{c}}$ & $7.55^{\mathrm{d}}$ & $6.14^{\mathrm{c}}$ \\
\hline
\end{tabular}

Means within a column having the different letter of superscript are significantly different from each other at 0.05 level of significance using Duncan test.

The genetic correlation coefficient generated by 20 ISSR markers was calculated using NTSYSpc 2.1. The results showed that there was a large variation among Ganoderma genotypes (Table 6). The lowest coefficient was found between CC and LD (0.44) whereas CC had the highest coefficient with LD (0.90).
Table 5. Characteristics of DNA profiles generated in nine Ganoderma genotypes by 20 ISSR primers.

\begin{tabular}{lllll}
\hline ISSR Primer & SB & NPB & PPB $(\%)$ & PIC \\
\hline UBC880 & 14 & 14 & 100 & 0.73 \\
UBC825 & 14 & 13 & 92.8 & 0.90 \\
UBC841 & 12 & 12 & 100 & 0.85 \\
UBC855 & 10 & 10 & 100 & 0.87 \\
UBC813 & 9 & 9 & 100 & 0.93 \\
UBC853 & 13 & 13 & 100 & 0.74 \\
UBC809 & 12 & 12 & 100 & 0.79 \\
UBC814 & 15 & 15 & 100 & 0.91 \\
UBC811 & 13 & 13 & 100 & 0.83 \\
UBC810 & 14 & 14 & 100 & 0.81 \\
UBC808 & 10 & 10 & 100 & 0.71 \\
UBC814- 11 & 14 & 14 & 100 & 0.85 \\
UBC818 & 12 & 12 & 100 & 0.85 \\
UBC820 & 13 & 13 & 100 & 0.77 \\
UBC826 & 10 & 10 & 100 & 0.83 \\
UBC826- 11 & 13 & 12 & 92.3 & 0.92 \\
UBC834 & 8 & 8 & 100 & 0.88 \\
UBC848 & 9 & 9 & 100 & 0.94 \\
UBC854 & 16 & 16 & 100 & 0.92 \\
UBC856 & 13 & 13 & 100 & 0.87 \\
Sum & 244 & 242 & - & - \\
Average & 12.2 & 12.1 & 99.25 & 0.85 \\
\hline SB: Scred
\end{tabular}

SB: Scored bands; NPB: number of polymorphic bands; PPB: percentage of polymorphic bands; PIC: polymorphism information content.

Based on the matrix of similarity coefficient, the genetic relatedness of nine Ganoderma genotypes was constructed and shown in Figure 4. The obtained dendrogram can be divided into two main groups. Group I consisted of four genotypes namely TG1, TG3, BD1, and $\mathrm{BD} 2$, while the remaining five genotypes were clustered in group II. The result of clustering analysis revealed that the studied Ganoderma spp. genotypes were not grouped based on geographical location where the samples were collected. This could be due to the exchanging of Ganoderma spp. producers, resulting in the same genotype having different names in different locations. Furthermore, the high diversity of collected samples in Vietnam could be due to other reasons such as out-crossing system or the geographical difference.

Table 6: Similarity coefficients among nine Ganoderma genotypes with 20 ISSR markers.

\begin{tabular}{llllllllll}
\hline & TG1 & TG2 & TG3 & LD & DN & CC & BD1 & BD2 & HN \\
\hline TG1 & 1.00 & & & & & & & & \\
TG2 & 0.59 & 1.00 & & & & & & \\
TG3 & 0.59 & 0.48 & 1.00 & & & & & \\
LD & 0.51 & 0.62 & 0.46 & 1.00 & & & & \\
DN & 0.52 & 0.58 & 0.51 & 0.60 & 1.00 & & & \\
CC & 0.50 & 0.61 & 0.51 & 0.90 & 0.68 & 1.00 & & \\
BD1 & 0.55 & 0.48 & 0.76 & 0.48 & 0.50 & 0.51 & 1.00 & 0.50 \\
BD2 & 0.57 & 0.50 & 0.80 & 0.44 & 0.52 & 0.49 & 0.77 & 1.00 \\
HN & 0.49 & 0.69 & 0.53 & 0.64 & 0.62 & 0.60 & 0.52 & 0.50 \\
\hline
\end{tabular}


In conclusion, nine Ganoderma accessions collected in Southern Vietnam show high variation in developmental and morphological characteristics, as well as in genetic compositions which implies the diverse gene pool in this region. This difference will be useful for Ganoderma breeding programs as the breeders could have more materials to expand the genetic base of new Ganoderma cultivars which is necessary to enhance the yield and quality. Furthermore, the distinguish DNA banding of each genotype could be used in molecular assisted-selection in breeding programs.

\section{ACKNOWLEDGEMENTS}

The authors express their deepest gratitude to the Faculty of Biotechnology, Ho Chi Minh City University of Food Industry, Vietnam for providing research facilities.

\section{REFERENCES}

Bao XF, Dong Q. Wang XS. 2002. Structural features of immunologically active polysaccharides from Ganoderma lucidum. Phytochemistry 59 (2): 175-181. DOI: 10.1016/S0031-9422(01)00450-2

Botstein D, White RL, Skolnick M, Davis RW. 1980. Construction of a genetic linkage map in man using restriction fragment length polymorphisms. Am J Hum Genet 32 (3): 314-331.

Du J, Guo HB, Li Q, Forsythe A, Chen XH, Yu XD. 2018. Genetic diversity of Lepista nuda (Agaricales, Basidiomycota) in Northeast China as indicated by SRAP and ISSR markers. PLoS ONE 13 (8): e0202761. DOI: 10.1371/journal.pone.0202761

Hong SG, Jung HS. 2004. Phylogenetic analysis of Ganoderma based on nearly complete mitochondrial small-subunit ribosomal DNA sequences. Mycologia 96 (4): 742-755. DOI: 10.2307/3762108

Iqbal A, Sadia B, Khan AI, Awan FS, Kainth RA, Sadaqat HA. 2010 Biodiversity in the sorghum (Sorghum bicolor L. Moench) germplasm of Pakistan. Genet Mol Res 9: 756-764. DOI: 10.4238/vol9-2gmr741

Jaipreet KR, Mohamamd A, Uma SS. 2015. Relative efficiency of RAPD and ISSR markers in assessment of DNA polymorphism and genetic diversity among Pseudomonas strains. Afr J Biotechnol 14 (13): 1097-1106. DOI: 10.5897/ajb10.1951

Jing Y, Peng M, Yang L, Wang Q. 2017. Evaluation of genetic diversity among Piptoporus betulinus as revealed by inter simple sequence repeat markers. Biotechnol Biotec Eq 31 (2): 333-338. DOI: 10.1080/13102818.2016.1276413

Le DHV, Tran DH. 2008. Biological characteristics and yield of some trains of lingzhi mushroom (Ganoderma lucidum) in Thue Thien Hue province. Hue Univ J Sci 49: 209-216. [Vietnamese]

Levi A, Thomas CE, Newman M, Reddy OUK, Zhang X, Xu Y. 2004. ISSR and AFLP markers differ among American watermelon cultivars with limited genetic diversity. J Am Soc Hortic Sci 129 (4): 553-558. DOI: 10.21273/jashs.129.4.0553

Liu SR, Ke BR, Zhang WR, Liu XR. Wu XP. 2017. Breeding of new Ganoderma lucidum strains simultaneously rich in polysaccharides and triterpenes by mating basidiospore-derived monokaryons of two commercial cultivars. Sci Hortic 216: 58-65. DOI: 10.1016/j.scienta.2016.12.016

Patel P, Rajkumar BK, Parmar P, Shah R, Krishnamurthy R. 2018. Assessment of genetic diversity in Colletotrichum falcatum Went accessions based on RAPD and ISSR markers. J Genet Eng Biotechnol 16 (1): 153-159. DOI: 10.1016/j.jgeb.2017.11.006

Porebski S, Bailey LG, Baum BR. 1997. Modification of a CTAB DNA extraction protocol for plants containing high polysaccharide and polyphenol components. Plant Mol Biol Rep 15 (1): 8-15. DOI: $10.1007 / \mathrm{bf} 02772108$

Rohlf F. 1987. NTSYS-pc: Microcomputer Programs for Numerical Taxonomy and Multivariate Analysis. Am Stat 41 (4): p330. DOI: $10.2307 / 2684761$

Staniaszek M, Marczewski W, Szudyga K, Maszkiewicz J, Czaplicki A, Qian G. 2002. Genetic relationship between Polish and Chinese strains of the mushroom Agaricus bisporus (Lange) Sing determined by the RAPD method. J Appl Genet 43: 43-47.

Wolfe AD, Liston A. 1998. Contributions of PCR-based methods to plant systematics and evolutionary biology. In: Soltis PS, Soltis DE, Doyle JJ (eds.). Molecular Systematics of Plants: DNA sequencing. Kluwer, New York, USA. DOI: 10.1007/978-1-4615-5419-6_2 